\title{
The value of triggered geriatrics and palliative care consults in hospital medicine during the COVID-19 response
}

\author{
Elizabeth H. Prsic ${ }^{1}$, Jennifer A. Ouellet ${ }^{2}$, James M. Lai ${ }^{2}$ \\ ${ }^{1}$ Palliative Care Program, Department of Internal Medicine, Yale School of Medicine, New Haven, CT, USA; ${ }^{2}$ Section of Geriatrics, Department of \\ Internal Medicine, Yale School of Medicine, New Haven, CT, USA \\ Correspondence to: Elizabeth H. Prsic, MD. Palliative Care Program, Department of Internal Medicine, Yale School of Medicine, 333 Cedar Street P.O. \\ Box 208028 New Haven, CT, 06520-8028, USA. Email: elizabeth.prsic@yale.edu.
}

Submitted Nov 19, 2020. Accepted for publication Jan 07, 2021.

doi: $10.21037 / \mathrm{apm}-20-2297$

View this article at: http://dx.doi.org/10.21037/apm-20-2297

Ensuring goal-concordant care for older adults with COVID-19 is an important challenge for hospitalist providers. While goal-concordant care is widely accepted as an important concept, prognostic uncertainty and the heavy burden of COVID-19 infections among hospitalized older adults create distinct challenges for clinicians caring for these patients. Further, the prioritization of health outcomes varies in older adults, along with what medical interventions they are willing to receive in order to achieve goals such as life prolongation, symptom control, and preservation of function (1). Appreciation of these concepts are central to providing goal-concordant care despite the many challenges imposed by the COVID-19 pandemic. Geriatric medicine and palliative care clinicians receive specific training in balancing future uncertainty with current clinical status, making them well poised to support clinical teams in making complex decisions for older adults hospitalized with COVID-19.

The importance of early goals of care and advance care planning conversations for older adults hospitalized with COVID-19 has been clearly outlined (2). Given prolonged respiratory failure and long hospitalizations, outcomes for mechanically ventilated older adults continue to evolve. Overall, the disease is characterized by high mortality among patients requiring intensive care, with worsening outcomes with increasing age (3). The longer-term clinical trajectories of non-mechanically ventilated but seriously ill older adults are not well characterized. However, worsened health trajectories in mobility, self-care, and cognition seem likely in a high proportion of patients. Variability is substantial in patient and family willingness to decline intensive care interventions, even in the face of serious illness. Geriatrics and palliative care consultants bring specialized training and experience navigating goals of care in medically complex older patients facing serious illness and supporting those who care for them.

The COVID-19 pandemic also poses significant limitations on communication between patients, caregivers, and their clinicians. Increased clinical volume, high patient acuity, restricted visitation of family and caregivers, and isolation precautions present limitations in carrying out inpatient goals of care discussions. Conversations between medical providers and patients or their surrogate decisionmakers often occur virtually. Due to illness or comorbidity, patients may be unable to participate in medical decision making. Patients coming from residential facilities often have had visitor restrictions in place and have been unable to see family members or surrogate decision makers for weeks or months before hospitalization. Clinical status may also decline quickly and unexpectedly due to COVID-19, necessitating rapid clarification of goals of care. Meanwhile, high medical floor census volumes with strained hospitalist staffing may impact the time available for clinicians to devote to critical but time intensive goals of care discussions. Finally, clinicians caring for hospitalized patients may practice outside of their usual scope of practice and feel particularly challenged addressing complex goals of care in critically ill older patients without specialized communication skills training. The COVID-19 pandemic offers an opportunity for specialists in geriatrics and palliative care to support overwhelmed clinicians, particularly those providing care outside their areas of 
expertise or comfort level, or for those managing large censuses.

In response to these challenges at Yale New Haven Hospital, the inpatient geriatric medicine and palliative care consult teams collaborated to provide targeted inpatient consultations for older patients with COVID-19. Consult leaders engaged representatives from hospitalist medicine, critical care teams, and academic medical services to identify areas of greatest geriatric and palliative care support needs in advance care planning. Given the perceived need for prompt and accurate ascertainment of goals of care on admission in a high-risk patient cohort and the potential benefit to the patient, family, and healthcare provider through facilitating clear lines of communication, we focused our initiative on hospitalized adults $\geq 65$ with COVID-19 in non-ICU settings. When determining the optimal model of delivery, we considered several factors. Prior research investigating the outcomes of early palliative care consultations in an intensive care setting found an increased likelihood for code status to change from full code to do-not resuscitate and observed reduced direct costs (4). While the goal of our intervention was to increase access to goal-concordant care rather than a specific code status outcome, we considered early palliative care discussions an important component in providing patients and families access to critical decisionmaking support. Secondly, we recognized the need to facilitate prompt consultation through the use of a virtual triggered consult model, that had been proposed as a method for improved efficiency and patient outcomes through early access to subspecialty care (5). With respect to triggered palliative care consults, recent data has also demonstrated its association with a higher rate of transition to DNR/DNI and with higher rates of transfer to hospice care compared with patients who did not receive palliative care consultation (6).

Consequently, we used a palliative care nurse coordinator to screen patients daily in non-ICU settings to identify those who met inclusion criteria for consultation, which included COVID-19 positive patients aged $\geq 65$. Patients aged 65-79 were staffed by the palliative care consult service, while those aged $\geq 80$ were staffed by the geriatrics consult service. After identifying the patients, the palliative care nurse coordinator notified the covering provider that a given patient met criteria for consultation by our combined geriatrics and palliative care consult service. With permission, the nurse coordinator placed an electronic consultation order. The goal of targeted consultation was two-fold: (I) to address goals of care and support communication between the patient, family/guardian, and primary team, and (II) to provide recommendations for symptom control including, but not limited to, dyspnea, delirium, and agitation. We performed consultations through electronic medical record review, telephone conversations with medical teams and nurses, telephone and video visits with patients, and telephone and video conversations with families or legal guardians/conservators. Formalized scripts were developed and reviewed together as a team to help guide conversations assessing patient values and goals and to make specific healthcare intervention recommendations.

Daily virtual meetings were held with the interdisciplinary team, including geriatric medicine and palliative care attending physicians, fellows, advance practice providers, palliative care social workers, palliative care chaplains, and a palliative care pharmacist. During meetings, the team reviewed patient, family and front-line clinician needs. We developed a daily multidisciplinary plan using available resources. Meetings also served as a mechanism to discuss difficult cases and guide ongoing development in building communication skills for clinicians, trainees, and nurses.

To date, the geriatric medicine and palliative care teams have consulted on over 100 hospitalized older adults with COVID-19, providing support and education to patients, caregivers and surrogate decision makers, primary medical teams, and trainees. With the support of palliative care social work, we helped families navigate complex care and placement decisions due to the pandemic. Palliative care chaplaincy encouraged patients and families to share life stories, values, and beliefs to find meaning and comfort in a time of crisis. Our team shared information between patients, families, and hospitalist providers, building empathy and meaning in patient interactions, especially in situations where patients were unable to communicate. Through regular communication with patients, caregivers and medical providers, we helped promote compassionate, goal-concordant care. The collaborative effort between geriatrics and palliative care consult services allowed pooling of resources, as well as structured multidisciplinary rounds to share clinical and operational expertise, support individual consult providers, and monitor workflow as the pandemic waxed and waned. Future studies will assess the impact of this initiative on patient outcomes, including ICU utilization, transition to do-not-resuscitate orders, transition to comfort measures, and patient experience. Our intervention focused on older patients due to the adverse outcomes observed in this population, as well as their unique set of psychosocial and decision-making needs. It 
is important, however, to acknowledge that other factors besides age, notably race and ethnicity, may also identify key populations at risk for disparities in palliative and geriatritic care services (7). These factors and potential interventions will need to be considered further in future research. We also seek to evaluate the experience of the primary medical and hospitalist teams, as well as resident and trainee educational impact. The initial widespread acceptance of this program at our institution, however, underscores the need for a continued structured, multidisciplinary approach in providing patients, families/guardians, and the front-line hospital medical floor teams support for developing early advanced care plans, that accurately reflect patients' specific needs and preferences.

\section{Acknowledgments}

A special thanks to the Geriatric and Palliative Care Consult Teams at Yale New Haven Hospital, and Rosemary CroninOzyck RN for her consult triage support.

Funding: None.

\section{Footnote}

Provenance and Peer Review: This article was a standard submission to the journal. The article has undergone external peer review.

Conflicts of Interest: All authors have completed the ICMJE uniform disclosure form (available at http://dx.doi. org/10.21037/apm-20-2297). The authors have no conflicts of interest to declare.

Ethical Statement: The authors are accountable for all aspects of the work in ensuring that questions related to the accuracy or integrity of any part of the work are appropriately investigated and resolved.

Open Access Statement: This is an Open Access article distributed in accordance with the Creative Commons
Attribution-NonCommercial-NoDerivs 4.0 International License (CC BY-NC-ND 4.0), which permits the noncommercial replication and distribution of the article with the strict proviso that no changes or edits are made and the original work is properly cited (including links to both the formal publication through the relevant DOI and the license). See: https://creativecommons.org/licenses/by-nc-nd/4.0/.

\section{References}

1. Fried TR, Tinetti M, Agostini J, et al. Health Outcome Prioritization as a Tool for Decision Making Among Older Persons with Multiple Chronic Conditions. Arch Intern Med 2011;171:1854-6.

2. Curtis JR, Kross EK, Stapleton RD. The Importance of Addressing Advance Care Planning and Decisions About Do-Not-Resuscitate Orders During Novel Coronavirus 2019 (COVID-19). JAMA 2020;323:1771-2.

3. Richardson S, Hirsch JS, Narasimhan M, et al. Presenting Characteristics, Comorbidities, and Outcomes Among 5700 Patients Hospitalized With COVID-19 in the New York City Area JAMA 2020;323:2052-9. Erratum in: JAMA 2020;323:2098.

4. Zalenski RJ, Jones SS, Courage C, et al. Impact of Palliative Care Screening and Consultation in the ICU: A Multihospital Quality Improvement Project. J Pain Symptom Manage 2017;53:5-12.e3.

5. Wachter RM, Judson TJ, Mourad M. Reimagining Specialty Consultation in the Digital Age: The Potential Role of Targeted Automatic Electronic Consultations. JAMA 2019;322:399-400.

6. Ma J, Chi S, Buettner B, et al. Palliative Care Consultation in the Medical ICU: A Cluster Randomized Crossover Trial. Crit Care Med 2019;47:1707-15.

7. Bassett MT, Chen JT, Krieger N. Variation in racial/ ethnic disparities in COVID-19 mortality by age in the United States: A cross-sectional study. PLoS Med 2020;17:e1003402.
Cite this article as: Prsic EH, Ouellet JA, Lai JM. The value of triggered geriatrics and palliative care consults in hospital medicine during the COVID-19 response. Ann Palliat Med 2021;10(2):970-972. doi: 10.21037/apm-20-2297 